Präv Gesundheitsf 2022 · 17:379-384 https://doi.org/10.1007/s11553-021-00895-0 Eingegangen: 15. März 2021

Angenommen: 6. Juli 2021

Online publiziert: 1. September 2021

๑) Der/die Autor(en) 2021

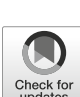

\section{Silke Heuse ${ }^{1}\left(\mathbb{D} \cdot\right.$ Uda-Mareke Risius $^{2}$}

'Fachbereich Wirtschaft und Psychologie, University of Europe for Applied Sciences, Hamburg, Deutschland

${ }^{2}$ Fachbereich Wirtschaft und Psychologie, University of Europe for Applied Sciences, Berlin, Deutschland

\title{
Stress bei Studierenden mit und ohne Nebenjob
}

\section{Welche Rolle spielen Stress-Mindsets?}

\begin{abstract}
Das Studium, die schönste Zeit im Leben? Oft ist das so, doch ist das Studieren auch durch viele Stressoren gekennzeichnet: Lernund Zeitdruck, Prüfungsängste, finanzielle Einschränkungen oder die Notwendigkeit, sich durch einen Nebenjob sein Studium zu finanzieren. Die vergangene Forschung zeigt, dass Studierende eine hohe Stressbelastung berichten. Gleichzeitig ist Stress negativ konnotiert und wird häufig als schädigend und als Risikofaktor dargestellt. Durch eine veränderte Sicht auf Stress, d. h. ein verändertes Mindset könnten nachteilige Effekte abgewendet werden.
\end{abstract}

\section{Stress bei Studierenden mit und ohne Nebenjob}

\section{Stress}

Stress ist ein vielfältig verwendeter Begriff, der im allgemeinen Verständnis mit einem Zustand der Angespanntheit und einer hohen Beanspruchung verbunden wird [19]. In der Forschung wird dieses Verständnis differenziert, indem zum einen die Stressreaktionen, zum anderen die Auslöser und Voraussetzungen für die Stressentstehung betrachtet werden [3, 19].

Die Erforschung von Stressreaktionen hat eine lange Tradition [3, 27] und gewann in der jüngsten Zeit durch die vermehrte Nutzung bildgebender Verfahren und interdisziplinärer Forschung zunehmend an Bedeutung. Es geht um die Fragen, welche Reaktionen durch andauernden Stress im Körper ausgelöst werden und welche Risiken für Erkrankungen damit einhergehen [24]. Dem gegenüber stehen Forschungsansätze, die konkrete Stressauslöser (z.B. Traumatisierungen, Katastrophen) und deren Auswirkungen auf den Menschen untersuchen. Für die Beeinflussung von Stresserleben stellt eine weitere Perspektive eine bedeutsame Grundlage dar: Das transaktionale Stressmodell [19] beschreibt, wie in Abhängigkeit von individuellen Bewertungen potenzieller Stressfaktoren (Ereigniseinschätzung) und der subjektiven Verfügbarkeit eigener Ressourcen (Ressourceneinschätzung) Stress entsteht. So können bei denselben äußeren Anforderungen in Abhängigkeit davon, was der einzelne diesen entgegenzusetzen vermag, unterschiedlich starke Stressbelastungen entstehen. Also können zwei Studierende, die sich auf dieselbe Prüfung vorbereiten, unterschiedlich viel Stress erleben, wenn der Eine meint, in einer unterstützenden Lerngruppe zu lernen und über einen guten Lernplan $\mathrm{zu}$ verfügen, während der Andere diese Ressourcen für sich nicht sieht.

\section{Stress von Studierenden}

Studierende sind mit einer Vielzahl von Stressoren konfrontiert [13] und erleben häufig ein hohes Ausmaß an Stress [2, 23]. Sie müssen zum einen mit studiumsbezogenen Anforderungen wie Prüfungsdichte, Stoffmenge und Zeitdruck [17] umgehen, zum anderen werden sie aber auch durch studiumsunabhängige Aufgaben wie Wohnortswechsel oder fa- miliäre Verpflichtungen [13]. Diese haben dann nicht nur Auswirkungen auf das Wohlbefinden, sondern auch auf die Leistungen der Studierenden [25]. Da eine Vielzahl von Studierenden auf einen Job neben dem Studium angewiesen ist, um finanziell unabhängig zu sein [16], soll in der vorliegenden Studie diese Tätigkeit als zusätzlich Stressquelle [10] berücksichtigt werden.

Entsprechend der transaktionalen Sichtweise auf Stress hängt das Ausmaß des erlebten Stresses von individuellen Ressourcen ab. Dies können z. B. soziale Unterstützungsquellen der Studierenden sein [23] oder ihre eigene Denk- und Sichtweise [17]. So geht eine höhere Selbstwirksamkeit, d.h. die Überzeugung, schwierige Situationen aufgrund der eigenen Kompetenzen bewältigen zu können, mit weniger Stresserleben einher [2].

\section{Stress-Mindsets}

Die Idee der Bewertung von Stress entsprechend des transaktionalen Verständnisses wird von der Stress-MindsetTheorie [9] aufgegriffen. Mindsets sind Überzeugungen oder implizite Theorien, die sich auf die Beurteilung von Situationen und das eigene Verhalten auswirken. So konnte auch bei Studierenden gezeigt werden, dass Überzeugungen hinsichtlich der eigenen Intelligenz und Persönlichkeit als angeboren und damit unveränderlich („fixed“) mit mehr Stress und schlechteren Leistungen einhergehen als solche, dass die eigene Intelligenz und Persönlichkeit durch Lernen („growth“) verändert werden kann [30]. Je nach 


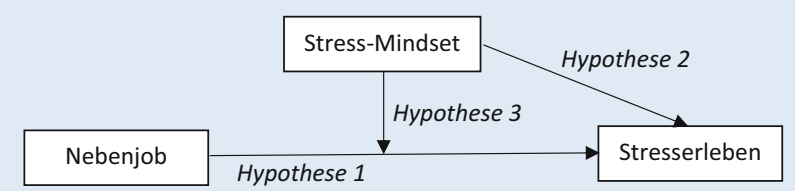

Abb. $1 \Delta$ Modell des Zusammenspiels von Stress-Mindsets, Stresserleben und Nebenjob

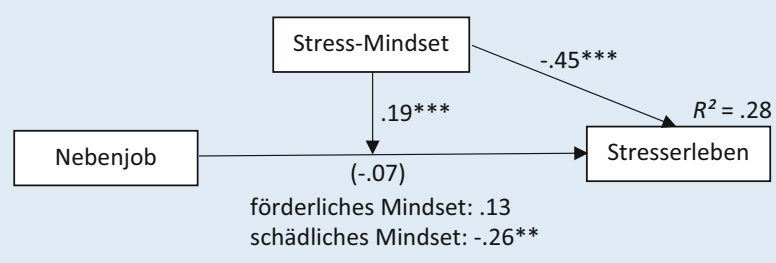

Abb. $2 \Delta$ Modell des Zusammenspiels von Stress-Mindsets, Stresserleben und Nebenjob (kontrolliert für Geschlecht; Nebenjob dichotom mit ja =1, nein $\left.=0 ;{ }^{* *} p<0,01,{ }^{* * *} p<0,001\right)$
Mindset unterscheiden sich Menschen darin, wie sie mit neuen Situationen und Herausforderungen umgehen. Mit der Stress-Mindset-Theorie wurde diese Idee auf das Verständnis von Stress als einerseits förderlich („enhancing“) oder andererseits schädlich („debilitating“) übertragen [8]. Das vorherrschende Stress-Mindset bestimmt, ob und welche physiologische und verhaltensbezogene Stressreaktionen auftreten [9]. Gleichzeitig schwächt die Überzeugung, dass Stress förderlich sein kann, die nachteiligen Auswirkungen von Stress auf Gesundheit und Leistungsfähigkeit ab [6]. Dieser Puffer konnte auch für Studierende gezeigt werden [14].

\section{Ziel der Studie}

Da viele Studierende zur Finanzierung ihres Studiums und ihres Unterhalts neben dem Studium einen Job nachgehen, stellt sich die Frage, ob diese von den Studierenden als Stressor wahrgenommen werden [13]. In Hypothese 1 wird daher postuliert:

H1. Eine Nebenjobtätigkeit geht mit einem erhöhten Stresserleben einher.

Über dieses Wissen um das Stresserleben von Studierenden hinaus soll die Rolle der Stress-Mindsets beleuchtet werden. Es gilt aufbauend auf dem bisherigen Wissen [9] herauszufinden, ob förderliche Stress-Mindsets auch mit weniger erlebten Stress der Studierenden einhergeht. Entsprechend wird in Hypothese 2 postuliert:

H2. Ein förderliches Stress-Mindset geht mit weniger Stress einher.
Aus der Idee der verschiedenen StressMindsets [9] leitet sich die ergänzende Frage, inwieweit Stress-Mindsets den Zusammenhang von Nebenjobtätigkeiten und Stresserleben der Studierenden weiter qualifizieren. In Hypothese 3 wird daher davon ausgegangen:

H3. Stress-Mindsets moderieren den Zusammenhang zwischen einer $\mathrm{Ne}$ benjobtätigkeit und dem Stresserleben derart, dass eine Nebenjobtätigkeit bei einer gleichzeitigen förderlichen Sicht auf Stress mit weniger Stresserleben assoziiert ist.

In Abb. 1 werden die Hypothesen modellhaft dargestellt.

\section{Methodik}

\section{Design und Stichprobe}

Diese Querschnittsstudie wurde an der University of Europe for Applied Sciences in Übereinstimmung mit den ethischen Grundsätzen der Deklaration von Helsinki durchgeführt. Nach aufgeklärter Einwilligung füllten 264 Studierende in Psychologie-, Wirtschafts- und künstlerischen Studiengängen einen Onlinefragebogen aus. In der vorliegenden Studie werden Daten von $N=195$ Studierenden mit vollständigem Datensatz ausgewertet, sie unterschieden sich in keiner der Studienvariablen von denjenigen mit fehlenden Daten $(p>0,05)$. Die Mehrheit der Studierenden $(79,0 \%)$ war zwischen 20 und 25 Jahre alt, nur 8,7\% waren jünger als 19 Jahre und 12,3\% waren älter als 26 Jahre. Zwei Drittel (67,2\%) waren weiblich, ein Drittel (32,8\%) männlich, das Geschlecht divers war nicht vertreten. Im Durchschnitt studierten die
Studierenden in ihrem $M=3,9$ Semester $(S D=1,8$, eine Angabe fehlte).

\section{Erhebungsinstrument}

Stress. Wahrgenommene Stressbelastungen der Studierenden wurden mit der deutschen Version [17] der Perceived Stress Scale (PSS-10; [4]) erfasst (z. B. „Wie oft hattest du dich nervös und ,gestresst' gefühlt?"). Die Antworten konnten auf einer 5-Punkt-Likert-Skala abgestuft werden ( $0=$ nie; $4=$ sehr oft). Die 10 Items wurden anschließend $\mathrm{zu}$ einem Summenwert zusammengefasst (Cronbach's $\alpha=0,86$ ), so dass höhere Werte für ein höheres Ausmaß an wahrgenommenem Stress mit einem möglichen Bereich zwischen 0 und 40 stehen.

Stress-Mindsets. Die Stress-Mindsets der Studierenden wurden mit dem General Stress Mindset Measure (SMM-G; [9]) in einer eigenen deutschen Version der Skala erfasst. Diese wurde gemäß gängiger Empfehlungen [28] für Instrumentenübersetzungen entwickelt. Die Skala besteht aus 8 Items, von denen jeweils 4 Items das Stress-Mindset „Stress ist förderlich“ (z.B. „Stress fördert bei mir das Lernen und meine Entwicklung.“) und das Stress-Mindset „Stress ist schädlich“ (z.B. „Gestresst zu sein schwächt meine Leistung und Produktivität.") erfassen. Die Antworten wurden ebenfalls auf einer 5-Punkt-Likert-Skala angegeben $(0=$ stimme gar nicht $\mathrm{zu}$; 4 = stimme sehr zu). Nach Umpolung der „Stress ist schädlich“-Items wurde ein Mittelwert gebildet (Cronbach's $\alpha=0,86$ ), so dass höhere Werte eine Denkweise 
repräsentieren, die Stress als förderlich wahrnimmt.

Nebenjob. Darüber hinaus wurden die Studierenden gefragt, ob sie einem $\mathrm{Ne}$ benjob nachgehen $(\mathrm{ja}=1 /$ nein $=0)$ und wie viele Stunden in der Woche.

\section{Datenauswertung}

Zunächst werden die Daten deskriptiv dargestellt und Geschlechtseffekte in den zentralen Studienvariablen mittels $\chi^{2}$ Tests und einfaktoriellen Varianzanalysen untersucht. Anschließend werden die Hypothesen mittels Pearson-Korrelation und Moderatoranalyse basierend auf multiplen Regressionsmodellen mit Hilfe des PROCESS-Makros (Version 2.041) überprüft [11]. Wie empfohlen [4], wurden der Prädiktor (Nebenjob; als Dummy-Variable) und Moderator (Stress-Mindset) zum Schutz vor Multikollinearität bei der Bildung des Interaktionsterms beider Variablen um ihre Mittelwerte zentriert. Im Fall einer signifikanten Interaktion wird der Effekt des Prädiktors auf das Kriterium auf verschiedenen Ebenen des Moderators $(M \pm 1 \mathrm{SD})$ bestimmt.

\section{Ergebnisse}

\section{Stressbelastungen von Studierenden}

Die Studierenden berichteten eine mittlere Stressbelastung mit $M=17,14$ ( $S D=$ $5,21)$ bei einem möglichen Wertebereich von $0-40$, wobei die Werte der weiblichen Studierenden etwas höher lagen als die der männlichen Studierenden (s. - Tab. 1).

Etwa zwei Drittel der Studierenden $(62,6 \%)$ gaben an, einem Nebenjob mit einer durchschnittlichen Wochenarbeitszeit von $M=15,5 \mathrm{~h}(S D=9,9)$ nachzugehen. Weder in der Nebentätigkeit $\left(\chi^{2}[1]=2,52 ; p>0,05\right)$, noch in der Wochenarbeitszeit $(F[1,121]=1,25 ; p>0,05)$ unterschieden sich die Geschlechter.

Entgegen Hypothese 1 unterschieden sich Studierende mit und ohne Nebenjob nicht signifikant in ihrer Stressbelastung (s. - Tab. 1) und auch die wöchentliche

Präv Gesundheitsf 2022 • 17:379-384 https://doi.org/10.1007/s11553-021-00895-0

c c Der/die Autor(en) 2021

\section{S. Heuse $\cdot$ U.-M. Risius}

\section{Stress bei Studierenden mit und ohne Nebenjob. Welche Rolle spielen Stress-Mindsets?}

\section{Zusammenfassung}

Hintergrund und Fragestellung. Studierende sind mit einer Vielzahl von Herausforderungen konfrontiert und erleben nicht selten ein hohes Maß an Stress. Wie kann das Verständnis von Stress-Mindsets, also verschiedenen Sichtweisen auf Stress, zur Reduktion von Stress beitragen? Dies soll die vorliegende Studie herausfinden. Als besondere Herausforderung wird dabei die Notwendigkeit vieler Studierender, durch einen Nebenjob ihr Studium zu finanzieren, berücksichtigt.

Methoden. Im Rahmen dieser Querschnittsstudie wurden $N=195$ Studierende mit einem Onlinefragebogen zu ihrem Stresserleben, Stress-Mindsets und Nebentätigkeit befragt. Die Auswertung erfolgte mittels Korrelationsund Moderationsanalysen.

Ergebnisse. Es zeigt sich ein erhöhtes Maß an Stresserleben der Studierenden.
Ein Mindset, dass Stress förderlich für die eigene Leistungsfähigkeit und Gesundheit sein kann, ging mit einem geringeren $\mathrm{Ma}$ an Stress einher. Nebenjobs erwiesen sich als Puffer für die negativen Auswirkungen eines schädlichen Stress-Mindsets auf das Stresserleben der Studierenden.

Schlussfolgerung. Stress ist aus dem Leben von Studierenden nicht wegzudenken. Daher ist es umso wichtiger zu wissen, wie Stress beeinflusst und genutzt werden kann. Mit einer Sichtweise, dass Stress förderlich ist, werden Stressoren als weniger aversiv erlebt. Dies sollten sich zukünftige Programme zur Stressreduktion von Studierenden zunutze machen.

Schlüsselwörter

Stresserleben - Stressmanagement .

Ressourcen $\cdot$ Risikofaktor $\cdot$ Bewertung

\section{Stress among students with and without part-time employment. What's the role of stress mindsets?}

Abstract

Background and objectives. Students are faced with a number of stressors. How can the knowledge of different stress mindsets, i.e. beliefs about enhancing or debilitating effects of stress, be used to reduce stress. As special stressor, students' part-time employment to supplement income while studying will be considered.

Methods. In this cross-sectional study, $N=195$ students completed an online questionnaire about their experience regarding stress, stress mindsets and parttime employment. Hypotheses were analysed by means of correlation and moderation analyses.

Results. Findings show that the students experience a medium amount of stress.

A mindset that stress can enhance one's own performance and health were related to less stress experiences. Part-time employment emerged as a buffer of detrimental effects of debilitating stress mindsets on students' stress experiences.

Conclusion. Stress is an integral part of student life. Hence, it is important to understand how to reduce or use stress experiences. Believing that stress is enhancing, stressors are experienced as less aversive. These findings have high implications for future stress reduction or stress management programs offered to students.

\section{Keywords}

Stress experience - Stress management . Resources · Risk factor · Appraisal
Arbeitszeit hing nicht mit der Stressbelastung zusammen (s. • Tab. 2).

\section{Stress-Mindsets von Studierenden}

Hinsichtlich der Bewertung von Stress tendieren die Studierenden zu einer negativeren Sichtweise, d.h. einem Mindset, das Stress als schädlichen Faktor für Leistung, Gesundheit und Wohlbefinden versteht $(M=1,48 ; S D=0,72)$. Hierbei zeigen sich keine Geschlechtsunterschiede und auch die Nebentätigkeit spielt keine direkte Rolle für die Ausprägungen der Stress-Mindsets. 


\section{Originalarbeit}

Tab. 1 Mittelwertvergleiche im Stresserleben und den Stress-Mindsets nach Geschlecht und

Nebentätigkeit

\begin{tabular}{|c|c|c|c|c|}
\hline & \multicolumn{2}{|c|}{ Geschlecht der Studierenden } & \multirow[t]{3}{*}{$F(1,194)$} & \multirow[t]{3}{*}{$p$} \\
\hline & Männlich & Weiblich & & \\
\hline & $M(S D)$ & $M(S D)$ & & \\
\hline Stresserleben & $15,39(5,50)$ & $18,00(4,86)$ & 11,36 & $<0,001$ \\
\hline \multirow[t]{4}{*}{ Stress-Mindset } & $1,58(0,78)$ & $1,44(0,69)$ & 1,59 & 0,21 \\
\hline & \multicolumn{2}{|c|}{ Nebentätigkeit } & \multirow[t]{3}{*}{$F(1,194)$} & \multirow[t]{3}{*}{$p$} \\
\hline & Ja & Nein & & \\
\hline & $M(S D)$ & $M(S D)$ & & \\
\hline Stresserleben & $16,81(4,90)$ & $17,70(5,69)$ & 1,33 & 0,25 \\
\hline Stress-Mindset & $1,52(0,76)$ & $1,43(0,66)$ & 0,76 & 0,39 \\
\hline
\end{tabular}

Tab. 2 Mittelwerte $(M)$, Standardabweichungen $(S D)$ und Interkorrelationen von Stresserleben, Stress-Mindset und wöchentlicher Arbeitszeit

\begin{tabular}{|c|c|c|c|c|}
\hline & $M$ & $S D$ & 2 & 3 \\
\hline 1. Stresserleben & 17,14 & 5,21 & $-0,45^{*}$ & $-0,01$ \\
\hline 2. Stress-Mindset & 1,48 & 0,72 & & $-0,08$ \\
\hline 3. Wochenarbeitszeit & 15,54 & 9,93 & & \\
\hline
\end{tabular}

Die Bedeutung der Mindsets wird sichtbar, wenn sie mit der tatsächlich wahrgenommenen Stressbelastung in Verbindung gebracht werden. Im Einklang mit Hypothese 2 berichteten die Studierenden, die Stress eher als aktivierend und förderlich ansahen, insgesamt weniger Stress (s. - Tab. 2). Der Geschlechtsunterschied in der Stressbelastung bleibt bestehen $(F[1,166]=7,44$; $p<0,001)$, wenn in einer ANCOVA für Stress-Mindsets kontrolliert wird.

Wie oben beschrieben hängt zwar die Nebentätigkeit nicht direkt mit dem wahrgenommenen Stress zusammen, doch kann sich dieser Zusammenhang unter verschiedenen Bedingungen ändern. Im Folgenden wurde daher untersucht, welche Rolle Stress-Mindsets hinsichtlich des Zusammenhangs zwischen einer Nebentätigkeit und Stresserleben spielen. Zur Überprüfung der Hypothese 3 wurde in einem Moderationsmodell das Stresserleben der Studierenden durch ihre Nebenjobtätigkeit, ihre StressMindsets sowie deren Interaktion vorhergesagt und für das Geschlecht der Studierenden kontrolliert. Neben den bekannten Haupteffekten weist die signifikante Interaktion von Nebenjob und Stress-Mindsets $(\beta=0,19 ; p<0,01)$ auf

\section{Diskussion}

Dies ist eine der ersten Studien, die die Auswirkungen von Stress-Mindsets auf das Stresserleben von Studierenden unter Berücksichtigung ihrer Nebentätigkeit untersucht. Die Ergebnisse weisen auf die Stressbelastung von Studierenden hin, zeigen aber auch die Abhängigkeit von den vorherrschenden Mindsets. So berichten Studierende, die Stress als förderlich wahrnehmen, weniger Stress. Gleichzeitig scheint die Nebentätigkeit eine zusätzliche Ressource der Studierenden darzustellen - insbesondere im Fall von Mindsets, in denen Stressbelastungen als schädlich bewertet werden.

Das in der vorliegenden Studie gezeigte Stresserleben ist konsistent mit früheren Studien [1, 22]. Geschlechtsunterschiede in der Stressbelastung sind aus der Literatur bekannt, sind häufig aber auf den subjektiven Bericht statt auf die tatsächliche körperliche Stressantwort zurückzuführen [12]. Dies scheint auf nachteilige kognitive Bewertungen der weiblichen Studierenden zurückzuführen zu sein. Zwar zeigen sich hinsichtlich der Sichtweise auf Stress in der vorliegenden Studie keine Geschlechtsunterschiede, doch sind diese entsprechenden Sichtweisen eher negativ ausgeprägt, d.h. Stress wird als schädlich wahrgenommen. Zukünftige Forschung sollte daher überprüfen, ob Geschlechtseffekte im Stresserleben durch die Überzeugung, Stress sei ein positiver Faktor, ausgeglichen werden können.

Mit Blick auf die Stress-Mindsets weisen die Studierenden eine eher negative Sicht auf Stress auf. Dies entspricht den Ergebnissen früherer Studien [9] und dem vorherrschenden Verständnis in der Gesellschaft. Aufklärungskampagnen zur Gesundheitsförderung und Angebote zur Stressreduktion (z. B. von Krankenkassen) kommunizieren meistens Stress als Risikofaktor und implizieren damit diese Sichtweise. Unbestritten stellt Stress einen Risikofaktor dar [20], doch sollte darüber nicht vergessen werden, dass Stress auch nützliche Facetten hat. Es ist zunächst eine natürliche Reaktion unseres Körpers, um uns leistungsfähig zu machen [3]. Für Studierende in Prüfungssituationen könnte 
Tab. 3 Moderatoranalyse des Zusammenhangs von Nebentätigkeit und Stresserleben (direkte Effekte) nach Ausprägung der Stress-Mindsets (konditionale Effekte)

\begin{tabular}{|c|c|c|c|c|c|}
\hline & B & SE & $\beta$ & $t$ & $p$ \\
\hline \multicolumn{6}{|l|}{ Direkte Effekte } \\
\hline Geschlecht & $-1,93$ & 0,70 & $-0,18$ & $-2,75$ & 0,01 \\
\hline Nebenjob & $-0,73$ & 0,67 & $-0,07$ & $-1,09$ & 0,28 \\
\hline Stress-Mindset & $-3,26$ & 0,45 & $-0,45$ & $-7,20$ & 0,001 \\
\hline Interaktion Nebenjob*Stress-Mindset & 2,86 & 0,98 & 0,19 & 2,92 & 0,01 \\
\hline$R^{2}$ & 0,28 & & & & \\
\hline \multicolumn{6}{|l|}{ Konditionale Effekte } \\
\hline "Stress ist schädlich"-Mindset & $-2,80$ & 0,94 & $-0,26$ & $-2,97$ & 0,01 \\
\hline "Stress ist förderlich“-Mindset & 1,34 & 1,00 & 0,13 & 1,33 & 0,18 \\
\hline
\end{tabular}

ein förderliches Stress-Mindset zur Folge haben, dass sie sich aufgrund ihrer akuten Stresssituationen für die Prüfung gewappnet und mit Energie ausgestattet fühlen, anstatt dass sie Angst haben zu versagen.

Wird die Nebentätigkeit der Studierenden als potenzielle Stressquelle betrachtet, konnte in der vorliegenden Studie entgegen Hypothese 1 kein direkter Effekt gefunden werden. Dies reiht sich ein in eine Vielzahl von inkonsistenten Befunde in Literatur [26]. Eine Nebentätigkeit kann Schlüsselkompetenzen wie Zeitmanagement und wirtschaftliches Denken fördern [29] und wertvolle Einblicke in den Berufsalltag gewähren, insbesondere bei fachnahen Jobs [16].

In Einklang mit Hypothese 2 konnte gezeigt werden, je positiver das StressMindset ausgeprägt ist, d.h. als je förderlicher der Stress bewertet wird, desto weniger Stress wird berichtet. Dies resultiert im transaktionalen Verständnis daraus, dass potenzielle Stressquellen als irrelevant bewertet werden, da keine Bedrohung von ihnen ausgeht [19]. Außerdem wird der Teufelskreis unterbrochen, in dem aus Angst vor Stress noch mehr Stress entsteht.

Entgegen der Annahme in Hypothese 3 zeigte sich bei nachteiligen StressMindsets weniger Stresserleben bei Studierenden mit Nebentätigkeit. Dies stützt die bereits diskutierte Annahme [13], dass Nebentätigkeiten auch mit zusätzlichen Ressourcen einhergehen können, die den Nachteil der schädlichen StressMindsets auf das Stresserleben ausglei- chen. Neben der Berücksichtigung der Stress-Mindsets sollten weiterführende Studien explizit danach fragen, wie die Nebentätigkeit erlebt wird, ob als zusätzliche Belastung oder als Ressource.

\section{Limitation}

Abschließend soll noch auf einige Einschränkungen und Möglichkeiten weiterführender Forschung eingegangen werden. Zur Operationalisierung der wahrgenommenen Stressbelastung der Studierenden wurde der PSS-10 $[5,18]$ herangezogen, welcher hauptsächlich kognitive Wertung zur Kontrolle des Stressors sowie Gefühle der Belastung erhebt. Die herausfordernde Wahrnehmung von Stressoren und körperliche Stresssymptome fehlen. Zwar handelt es sich bei den Daten um Selbstberichte, die das Risiko von Verzerrungen (z.B. Geschlechtseffekten) bergen [12], doch gilt der PSS-10 als ein weit verbreitetes und valides Messinstrument [21]. Zukünftige Forschung sollte dennoch zwischen Stressoren, deren Bewertung und den ausgelösten Reaktionen differenzieren [7] und die Effekte der Stress-Mindsets von Studierenden weiter durch objektive Stressdaten sowie weitere Kriterien wie Wohlbefinden, Leistungsfähigkeit und Studienerfolg untermauern. Wie erwähnt, sollte auch die Erfassung der Nebentätigkeit in zukünftigen Studien ausdifferenziert werden, indem zwischen fächernahen und -fernen Jobs unterschieden wird und die individuel- len Nutzen und Kosten des Nebenjobs erfasst werden.

$\mathrm{Da}$ es sich bei der vorliegenden Studie um eine Querschittsstudie handelt, sollten die Effekte und Wechselwirkungen von Stress-Mindsets mit studiumspezifischen Stressoren (vgl. [13]) auch im Längsschnitt untersucht werden. Hier gibt es bereits interessante Interventionen, die Stress-Mindsets verändern und in entsprechende Studien eingebaut werden können [15]. Damit böten sie den Studierenden eine gute Basis für einen erfolgreichen Umgang mit Stress im Studium sowie eine bessere Vereinbarkeit von Studium und Nebenjob.

\section{Ausblick}

Die vorliegende Studie ergänzt das bisherige Wissen über die Stressbelastung von Studierenden und den Einfluss von Stress-Mindsets auf das Stressgeschehen, indem die Notwendigkeit eines Nebenjobs als relevanter studienspezifischer Faktor berücksichtigt wird. Die Erkenntnis, dass förderliche StressMindsets bei Studierenden mit weniger Stress einhergehen und dass die nachteiligen Effekte schädlicher Stress-Mindsets durch mögliche Ressourcen eines $\mathrm{Ne}$ benjobs abgepuffert werden können, sollten für die Arbeit mit Studierenden genutzt werden und zu differenzierten Sichtweisen auf Nebenjobs anregen. Die Aufklärung über und Veränderung von Stress-Mindsets sollte im Rahmen von Schlüsselkompetenzen Thema im Studium sein sowie als Angebote zur Studierendengesundheit dienen. In dieser Arbeit mit den Studierenden sollte eine Ausdifferenzierung von individuellen Stressoren (z. B. Prüfungsangst) genutzt werden, um personalisierte Angebote zu ermöglichen. Lehrende und Studierendenberatungen sollten diese Möglichkeit zur Stressreduktion von Studierenden kennen und nutzen.

\section{Fazit für die Praxis}
- Studierende sind mit einer Vielzahl von Anforderungen und Belastungen konfrontiert, so dass Stress zu ihrem Studienalltag dazugehört.




\section{- Stress-Mindsets stellen Überzeu- gungen dar, die Stress entweder als förderlich oder als schädlich für die eigene Leistungsfähigkeit und Gesundheit bewerten. \\ - Viele Studierende sind gezwungen, zusätzlich zum Studium durch einen Nebenjob ihr Studium und ihren Unterhalt zu finanzieren. Doch nicht immer ist diese Tätigkeit nachteilig. \\ - Wird Stress als förderlich wahrge- nommen, berichten Studierende per se weniger Stress.}

\section{Korrespondenzadresse}

\section{Silke Heuse}

Fachbereich Wirtschaft und Psychologie, University of Europe for Applied Sciences Museumstr. 39, 22765 Hamburg, Deutschland silke.heuse@ue-germany.com

Funding. Open Access funding enabled and organized by Projekt DEAL.

\section{Einhaltung ethischer Richtlinien}

Interessenkonflikt. S. Heuse und U.-M. Risius geben an, dass kein Interessenkonflikt besteht.

Im gesamten Forschungsablauf wurden die ethischen Richtlinien der Deklaration von Helsinki beachtet.

Open Access. Dieser Artikel wird unter der Creative Commons Namensnennung 4.0 International Lizenz veröffentlicht, welche die Nutzung, Vervielfältigung, Bearbeitung, Verbreitung und Wiedergabe in jeglichem Medium und Format erlaubt, sofern Sie den/die ursprünglichen Autor(en) und die Quelle ordnungsgemäß nennen, einen Link zur Creative Commons Lizenz beifügen und angeben, ob Änderungen vorgenommen wurden.

Die in diesem Artikel enthaltenen Bilder und sonstiges Drittmaterial unterliegen ebenfalls der genannten Creative Commons Lizenz, sofern sich aus der Abbildungslegende nichts anderes ergibt. Sofern das betreffende Material nicht unter der genannten Creative Commons Lizenz steht und die betreffende Handlung nicht nach gesetzlichen Vorschriften erlaubt ist, ist für die oben aufgeführten Weiterverwendungen des Materials die Einwilligung des jeweiligen Rechteinhabers einzuholen.

Weitere Details zur Lizenz entnehmen Sie bitte der Lizenzinformation auf http://creativecommons.org/ licenses/by/4.0/deed.de.

\section{Literatur}

1. Acharya L, Jin L, Collins W (2018) College life is stressful today-emerging stressors and depressive symptoms in college students. J Am Coll Health 66:655-664

2. Büttner TR, Dlugosch GE (2013) Stress im Studium. Präv Gesundheitsf 8:106-111

3. Cannon WB (1932) The wisdom of the body Norton, New York

4. Cohen J, Cohen P, West SG, Aiken LS (2002) Applied multiple regression/correlation analysis for the behavioral sciences. Routledge, New York

5. Cohen S, Kamarck T, Mermelstein R (1983) A global measure of perceived stress. J Health Soc Behav 24:385-396

6. Crum AJ, Akinola M, Martin A, Fath S (2017) The role of stress mindset in shaping cognitive, emotional, and physiological responses to challenging and threatening stress. Anxiety Stress Coping 30:379-395

7. Crum AJ, Jamieson JP, Akinola M (2020) Optimizing stress: an integrated intervention for regulating stress responses. Emotion 20:120-125

8. Crum AJ, Lyddy C (2013) De-stressing stress: the power of mindsets and the art of stressing mindfully. In: Ngnoumen C, le A, Langer E (Hrsg) The Wiley Blackwell handbook of mindfulness. Wiley-Blackwell, Chichester, S948-963

9. Crum AJ, Slovey P, Achor S (2013) Rethinking stress: the role of mindsets in determining the stress response. J Pers Soc Psychol 104:716-733

10. Curtis S, Williams J (2002) The reluctant workforce: undergraduates' part-time employment. Educ Train 44:5-10

11. Hayes AF (2013) Methodology in the social sciences. Introduction to mediation, moderation, and conditional process analysis: a regressionbased approach. Guilford, New York

12. Helbig S, Backhaus J (2017) Sex differences in a real academic stressor, cognitive appraisal and the cortisol response. Physiol Bahav 179:67-74

13. Heuse S, Dietze C, Fodor D, Voltmer E (2020) Studying and more: part-time employment as an educational challenge? J Med Psychol 22:59-65

14. Huebschmann NA, Sheets ES (2020) The right mindset: stress mindset moderates the association between perceived stress and depressive symptoms. Anxiety Stress Coping 33:248-255

15. Jamieson JP, Crum AJ, Goyer JP, Marotta ME, Akinola M (2018) Optimizing stress responses with reappraisal and mindset interventions: an integrated model. Anxiety Stress Coping 31:245-261

16. Kettner $P$ (2019) Belastung oder Bereicherung? Perspektiven Studierender auf das komplexe Verhältnis von Studium und (Neben-)Job. In: Richter S, Frieberthäuser B (Hrsg) Studieren Forschen - Praxis, Bd. 20. Goethe-Universität, Frankfurt, S179-194

17. Kirsch A-S, Laemmert P, Tittlbach S (2017) Gesundheitliche Anforderungen und Ressourcen von Studierenden. Präv Gesundheitsf 12:181-188

18. Klein EM, Brähler $E$, Dreier $M$, Reinecke $L$, Müller KW, Schmutzer G, Wölfing K, Beutel ME (2016) The German version of the perceived stress scale-psychometric characteristics in a representative German community sample. BMC Psychiatry 16:159-168

19. Lazarus R, Folkman S (1984) Stress, appraisal, and coping. Springer, New York

20. Lederbogen F, Ströhle A (2012) Stress, psychische Erkrankungen und koronare Herzkrankheit. Nervenarzt 83:1448-1457

21. Lee E-H (2012) Revise of the psychometric evidence of the perceived stress scale. Asian Nurs Res 6:121-127
22. Leppink EW, Odlaug BL, Lust K, Christenson G, Grant JE (2016) The young and the stressed: stress, impulse control, and health in college students. JNerv Ment Dis 204:931-938

23. Lutz-Kopp C, Meinhardt-Injac B, Luka-Krausgrill U (2018) Psychische Belastungen Studierender. Präv Gesundheitsf 14:256-263

24. McEwen BS (1998)Protectiveand damaging effects of stress mediators. NEngl J Med 338:171-179

25. RochfordC, ConnollyM, Drennan J (2009) Paid parttime employment and academic performance of undergraduate nursing students. Nurse Educ Today 29:601-606

26. Ryan M, Barns A, McAuliffe D (2011) Part-time employment and effects on Australian social work students: a report on a national study. Aust Soc Work64:313-329

27. Selye H (1936) A syndrome produced by diverse nocuous agents. Nature 138:32

28. Sousa VD, Rojjanasrirat W (2011) Translation, adaptation and validation of instruments or scales for use in cross-cultural health care research: a clear and user-friendly guideline. J Eval Clin Pract 17:268-274

29. Tully C, van Santen E(2020) Nebenjob. In:Bollweg $P$, Buchna J, Coelen T, Otto HU (Hrsg) Handbuch Ganztagsbildung. SpringerVS, Wiesbaden

30. Yager DS, Dweck CS (2012) Mindset that promote resilience: when students believe that personal characteristics can be developed. Educ Psychol 47:302-314 